\title{
Stability and Convergence Analysis for Different Harmonic Control Algorithm Implementations
}

\author{
Ilias Zazas and Steve Daley \\ Institute of Sound and Vibration Research, University of Southampton, Highfield, \\ Southampton, SO17 1BJ
}

\begin{abstract}
In many engineering systems there is a common requirement to isolate the supporting foundation from low frequency periodic machinery vibration sources. In such cases the vibration is mainly transmitted at the fundamental excitation frequency and its multiple harmonics. It is well known that passive approaches have poor performance at low frequencies and for this reason a number of active control technologies have been developed. For discrete frequencies disturbance rejection Harmonic Control (HC) techniques provide excellent performance. In the general case of variable speed engines or motors, the disturbance frequency changes with time, following the rotational speed of the engine or motor. For such applications, an important requirement for the control system is to converge to the optimal solution as rapidly as possible for all variations without altering the system's stability. For a variety of applications this may be difficult to achieve, especially when the disturbance frequency is close to a resonance peak and a small value of convergence gain is usually preferred to ensure closed-loop stability. This can lead to poor vibration isolation performance and long convergence times. In this paper, the performance of two recently developed HC algorithms are compared (in terms of both closed-loop stability and speed of convergence) in a vibration control application and for the case when the disturbance frequency is close to a resonant frequency. In earlier work it has been shown that both frequency domain HC algorithms can be represented by Linear Time Invariant (LTI) feedback compensators each designed to operate at the disturbance frequency. As a result, the convergence and stability analysis can be performed using the LTI representations with any suitable method from the LTI framework. For the example mentioned above, the speed of convergence provided by each algorithm is compared by determining the locations of the dominant closed-loop poles and stability analysis is performed using the open-loop frequency responses and the Nyquist criterion. The theoretical findings are validated through simulations and experimental analysis.
\end{abstract}

\section{Introduction}

The development of different approaches for the mitigation of low frequency machinery vibration has been the subject of intensive research in recent decades. Is such cases, the disturbances, are often produced by rotating machinery and the resulting noise or vibration spectra is dominated by multiple steady-state tones associated with the rotational speed of the machinery. Due to the poor performance of passive approaches at low frequencies (Elliott and Nelson, 1993) a number of active control technologies have been proposed and developed. The performance of an active control system mainly depends on the algorithm used to generate the appropriate control signals, based on information provided by a number of error sensors (i.e. accelerometers, microphones). Depending on the complexity of the system to be controlled, different strategies can be adopted; these include frequency or time-domain approaches where the controller can have a fixed parameter compensator form or be an update law. For the case of discrete frequency disturbance rejection, Harmonic Control (HC) has been shown to have excellent performance 
(Daley and Zazas, 2012; Daley et al., 2008; Hätönen et al., 2006). A discrete-time version of HC was first implemented by Shaw et al. (1989), while Hall and Wereley (1989) showed that for a single input, single output (SISO) system, the harmonic cancelation problem is similar to classical periodic disturbance rejection. A key result of their work was to show that the typical adaptive feedforward harmonic algorithm can be represented as a classical feedback compensator designed to cancel steady-state periodic disturbances of specific frequency $\omega_{o}$. Similar discrete-time compensator representations of adaptive feedforward algorithms for periodic disturbance cancellation have also been developed by Glover (1977), Widrow and Stearns (1985) and Elliott et al., (1987); however, these were based on different versions of least mean squares (LMS) algorithms rather than harmonic control.

In its standard form, the $\mathrm{HC}$ approach is implemented using a frequency domain steady-state approach where, following each corrective control action, the algorithm waits for transients to die out before executing the next update. In practice this can lead to significant convergence delays especially for the general case of variable speed machines where, the disturbance frequency changes with time and the controller is required to converge to the optimum solution as rapidly as possible following any variation and without altering the system's stability. In a similar manner, the presence of resonant peaks close to the disturbance frequencies can also lead to long convergence times as a small value of the convergence gain is usually adopted to ensure closed-loop stability.

In this paper two recently proposed $\mathrm{HC}$ algorithms (implemented in the frequency domain) are considered (Daley and Zazas, 2012; Daley et al., 2008). The first is referred to as the 'Instantaneous Harmonic Control' algorithm (IHC) while the second as the 'Recursive Least Squares based IHC' algorithm (RLS-IHC). Both operate in an instantaneous manner where the control input is updated at every time instant thereby accelerating the control action. Initial results (Zazas, 2009) indicated that for small values of the convergence gain, both algorithms converge to the noise floor at the same rate and they both provide similar levels of disturbance rejection. In addition, it was observed that when the disturbance frequency is close to a resonance peak, the RLS-IHC implementation provides a better stability margin, thereby allowing the value of the convergence gain to be increased, which in turns results in a faster transient response. Although both of the above observations are true, a mathematical derivation to explain the stability and convergence behaviour of both algorithms could not be derived since the overall controller is an update law rather than a fixed parameter compensator. The stability and convergence properties of both frequency domain algorithms could only be examined on a trial and error basis, where the algorithms' parameters are tuned (either online or through simulations) until the system's output reaches the optimum solution. Such a methodology, apart from being time consuming and dependant on a very accurate model of the process, cannot provide a mathematical explanation of the algorithms' convergence behaviour and stability robustness.

Recently it was shown that both algorithms presented herein, can be represented / approximated as Linear Time Invariant (LTI) fixed parameter compensators (Hätönen et al., 2006; Zazas, 2009), thereby enabling the stability and convergence properties of both implementations to be examined through classical LTI theory. It is well known for example, that the transient response of a system is related to the locations of the closed-loop dominant poles (Kuo, 1991; Mulligan, 1949). Sievers and Von Flotow, (1992) for instance, using the equivalent Single Input - Single Output (SISO) LTI transfer function representation of the well-known Multiple Error LMS algorithm (Elliott et al., 1987) derived an expression for the algorithm's transient response, by determining the locations of the closed-loop system's poles for different values of the convergence gain. The transient behaviour of the IHC algorithm has recently been examined by Orivuori and Zenger (2012) who compared the performance of different linear control algorithms for the active control of tonal disturbances. However, the comparison is based purely on time-domain simulations and no analytical expressions are derived to formally explain the transient behaviour of each control methodology considered.

In this paper, a similar approach to that used by Sievers and Von Flotow, (1992) is adopted in an attempt to theoretically explain the transient response and closed loop stability characteristics of both the 
IHC and the RLS-IHC algorithms. More specifically, the LTI representations of both frequency domain $\mathrm{HC}$ algorithms are utilised to derive a mathematical expression for the location of the dominant closedloop poles and relate these to their respective transient responses. It will be shown that the derived expressions do indeed explain the similar transient behaviour that is observed when the disturbance frequency is far from a resonance peak or when a small value of the convergence gain is used; a result that is also confirmed through simulations and experimental validation. In addition it will be seen that the fact that the RLS based fixed parameter compensator has been derived as a combination of two individual band-pass filters, both centred at the disturbance frequency, significantly improves the closed-loop system's stability margins. This is especially true in the stop-band regions of the open-loop frequency response where a resonant peak is more likely to affect system stability. This is an important result as the convergence gain can be further increased to improve the system's transient response and vibration isolation performance.

The paper is structured as follows: Section 2 describes the disturbance cancellation problem and how the classical HC approach can be adopted to tackle this problem. Section 3 presents the proposed HC algorithms together with their LTI fixed parameter representations. In Section 4 the closed-loop poles locations and the time constants for both cases are derived. In Section 5 the stability and convergence behaviour of the closed-loop system (for both compensator implementations) in terms of the convergence gain are examined by simulations and by using LTI techniques such as the open-loop frequency response, Nyquist plots and closed-loop pole locations. The experimental rig used for this exercise is described at the beginning of the section. Section 6 presents experimental results which verify the theoretical findings and conclusions are presented in Section 7.

\section{Disturbance Cancellation Problem and Harmonic Control}

Fig. 1 illustrates the disturbance cancellation problem; where $G_{c}(q)^{\dagger}$ is a transfer function representing a control path model (expressed in terms of the backward shift operator $q^{-1}$ ) and combines both the plant and actuator dynamics. This can be used to suppress a disturbance signal $d_{t}$ that acts through a disturbance path $G_{d}(q)$. It is assumed that $d_{t}$ is a sinusoid i.e. $d_{t}=P \cos \left(\omega_{o} t+\varphi\right)$, for some $P, \omega_{o}$ and $\varphi$, and that both transfer functions are stable, controllable and observable, and that only the signal $e_{t}=G_{c}(q) u_{t}+G_{d}(q) d_{t}$ is available for feedback control. Taking these assumptions into account, it is clear that the control objective is to find a controller that generates asymptotically, an input $u_{t}$ that satisfies

$$
e_{t}=G_{c}(q) u_{t}+G_{d}(q) d_{t}=0
$$

The fundamental idea behind $\mathrm{HC}$ is the well-known fact that the output of a Linear Time-Invariant (LTI) system subject to a sinusoidal excitation is a sinusoid of the same frequency but with modified amplitude and phase. Hence, because the disturbance signal it is assumed to be a sinusoid of frequency $\omega_{o}$ , it follows that the input signal that satisfies Eq.(1) is also a sinusoid of frequency $\omega_{o}$. Furthermore in HC, a frequency domain 'steady state' approach is usually adopted; therefore the plant (Eq.(1)) can be represented as:

\footnotetext{
${ }^{\dagger}$ A Single Input - Single Output (SISO) system is considered for ease of exposition.
} 


$$
e\left(e^{j \omega_{o} T}\right)=G_{c}\left(e^{j \omega_{o} T}\right) u\left(e^{j \omega_{o} T}\right)+G_{d}\left(e^{j \omega_{o} T}\right) d\left(e^{j \omega_{o} T}\right)
$$

where $T$ is the sampling period. Under the assumption that it is possible to extract a frequency domain estimate of the system's output $\hat{e}\left(e^{j \omega_{o} T}\right)$ for a given frequency $\omega_{o}$, from the original time domain signal $e_{t}$ different control algorithms can be developed. If for example, $G_{d}\left(e^{j \omega_{o} T}\right)$ was known and $d\left(e^{j \omega_{o} T}\right)$ could be measured, a feed-forward solution for $u\left(e^{j \omega_{o} T}\right)$ can be obtained by solving Eq.(2) for $e\left(e^{j \omega_{o} T}\right)=0$. However, in most applications this is not the case and a number of iterative feedback solutions have been proposed (Elliott and Nelson, 1993; Elliott et al., 1987). Using a gradient descent approach to minimise the error signal for example, the following 'steady-state' algorithm for recursively updating the control input can be derived

$$
u_{k}\left(e^{j \omega_{o} T}\right)=\alpha \cdot u_{k-1}\left(e^{j \omega_{o} T}\right)-\beta \cdot C \cdot \hat{e}_{k-1}\left(e^{j \omega_{o} T}\right)
$$

Where $k$ represents an iteration index, $\alpha$ is a leakage gain $(0<\alpha \leq 1), \beta>0$ is the convergence gain, $C$ is a complex number (or matrix for the MIMO case), usually chosen to be either the inverse $G_{c}\left(e^{j \omega_{o} T}\right)^{-1}$, or the Hermitian transpose $G_{c}\left(e^{j \omega_{o} T}\right)^{H}$ of the plant evaluated at the disturbances frequency ${ }^{\ddagger}$. The embellishment $\hat{i}$ denotes estimate of $i$.

In $\mathrm{HC}$ it is quite common to use a sinusoid at the disturbance frequency (with unity amplitude) as a reference signal and this can be generated within the controller or from a tachometer signal (this is denoted as $z_{t}$ in Fig. 1). For this reason in general, harmonic control strategies can be considered as feedforward methods since this reference signal is filtered in such a way that the resulting sinusoid (at the control path output) matches in amplitude but is $180^{\circ}$ out of phase with the disturbance path signal, resulting in zero vibration transmission.

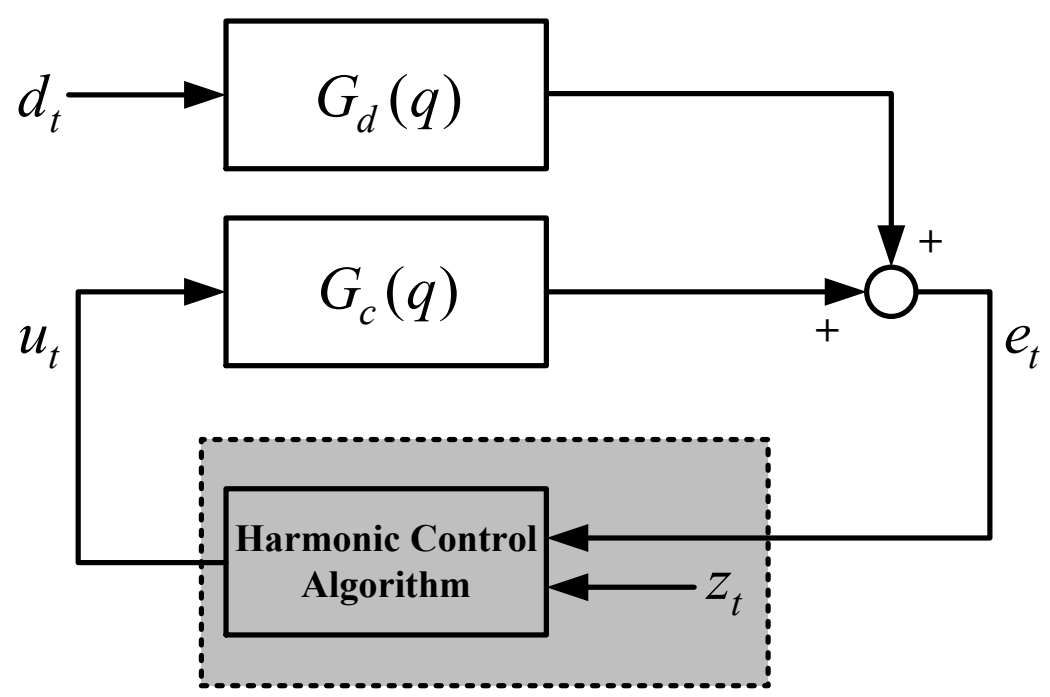

Figure 1: Vibration Control Problem.

\footnotetext{
$\$$ For the SISO system considered here these two are effectively equivalent.
} 


\section{Proposed HC algorithms and Equivalent LTI representations}

Both implementations of HC presented here use the same gradient descent approach, described by Eq.(3) to update the control input in the frequency domain. They differ though in the way the frequency domain estimate of the system's output $\hat{e}\left(e^{j \omega_{o} T}\right)$ is obtained. Fig. 2 shows a schematic diagram of both frequency domain implementations. The first, named as Instantaneous Harmonic Control (IHC) algorithm, (top diagram of Fig. 2) uses a discrete time Fourier decomposition to extract the gain and phase information from the time domain signal and construct the frequency domain estimate. The second (bottom diagram of Fig. 2), named as Recursive Least Squares based IHC algorithm (RLS-IHC), uses the well-known RLS algorithm for the same purpose (Daley and Zazas, 2012).
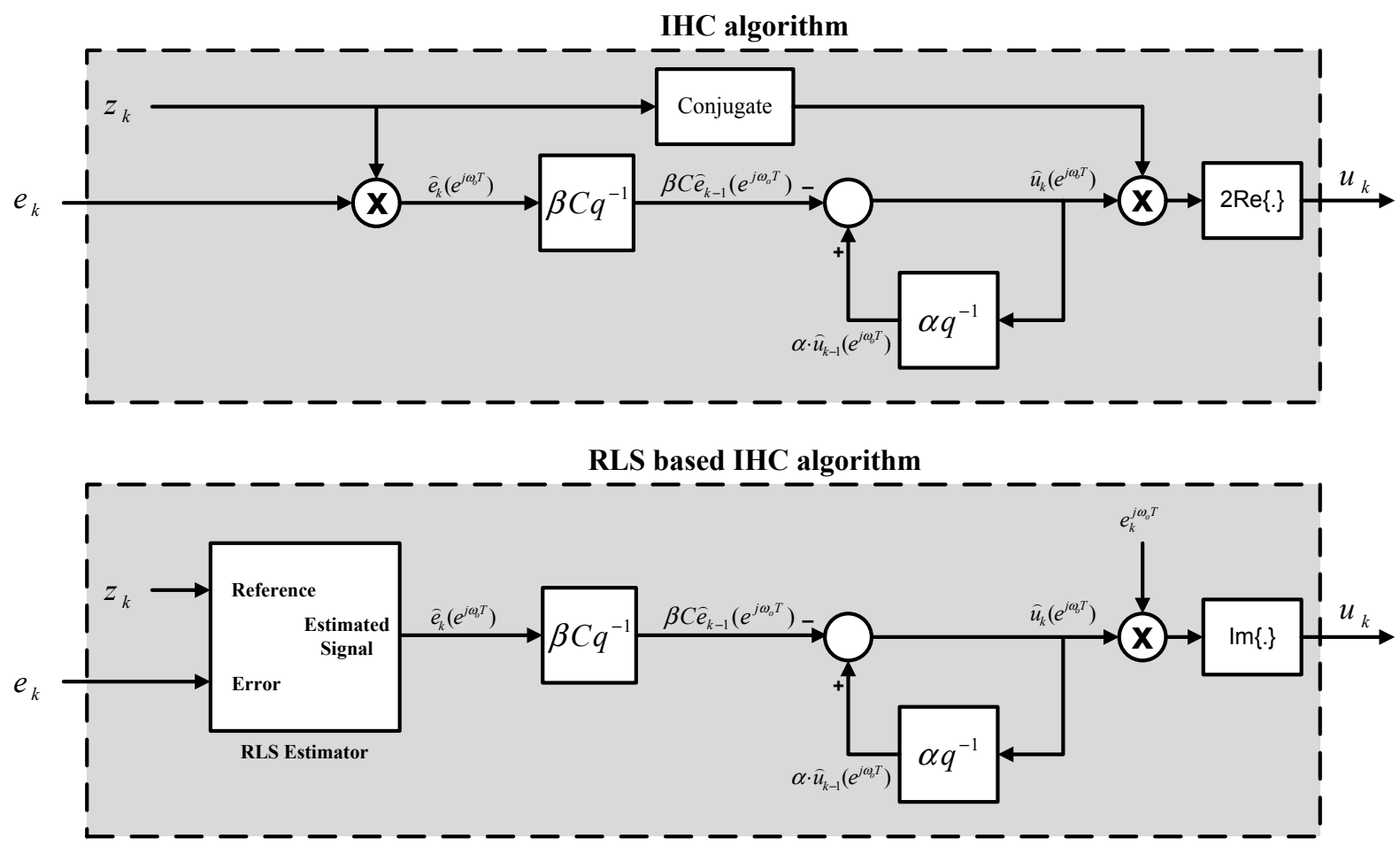

Figure 2: Harmonic control algorithms; IHC (top) and RLS based IHC (bottom).

In (Daley and Zazas, 2012; Daley et al., 2008; Zazas, 2009) it has been shown that both HC algorithms can be approximated as LTI feedback compensators given by Eq.(4) for the IHC algorithm and Eq.(5) for the RLS based IHC algorithm. The analysis is based on a SISO implementation and for a single tone disturbance. The methods however, can easily be extended for both the multi-harmonic and multivariable cases.

$$
\begin{gathered}
K_{I H C}(z)=-2 \beta A\left[\frac{\cos \left(\omega_{o} T+\phi\right) z-\alpha \cos (\phi)}{z^{2}-2 \alpha \cos \left(\omega_{o} T\right) \mathrm{z}+\alpha^{2}}\right] \\
K_{R L S}(z)=G_{R L S}(z) \cdot K_{I H C}(z)
\end{gathered}
$$

Where $A e^{j \phi}=C$ and $A e^{-j \phi}=C^{*}$ are the complex number of Eq.(3) and its conjugate respectively, $T$ is the sampling period and $G_{R L S}(z)$ is an LTI fixed parameter filter describing the RLS estimator and is given by: 


$$
G_{R L S}(z)=\frac{2(1-\lambda) \cdot\left(z^{2}-\cos \left(\omega_{o} T\right) z\right)}{(2-\lambda) z^{2}-2 \cos \left(\omega_{o} T\right) z+\lambda}
$$

where $\lambda$ is a forgetting factor (Zazas, 2009)]. In (Daley and Zazas, 2012; Zazas, 2009) it has been shown that the accuracy of the RLS fixed filter approximation increases monotonically with respect to the forgetting factor. Moreover, from Eq. (6) it is clear that the approximation is not valid for $\lambda=1$ since the gain term $2(1-\lambda)$ becomes zero. However, in practical application the forgetting factor will always be less than unity to enable the adaptive capabilities of the algorithm since during convergence the error response will be continuously varying. The selection of the forgetting factor therefore represents a compromise between the accuracy of the fixed filter approximation and the need to track changes in the error response. In addition, the value of the forgetting factor is closely related with the bandwidth of the RLS-fixed parameter filter Eq. (6). More specifically the bandwidth narrows as $\lambda$ increases as shown in Fig. 3. As a consequence the forgetting factor acts as a bandwidth regulator for the overall RLS-IHC compensator. Observing Eq. (5) it is then clear that the frequency domain implementation of the RLSIHC algorithm is equivalent to the combination of two individual band-pass filters both centred at the frequency of the disturbance signal, with the first (RLS fixed parameter filter) acting as an overall filter bandwidth regulator (Daley and Zazas, 2012). It will be shown in Section 5 that the additional filtering action introduced by the RLS fixed filter significantly improves the system's closed-loop stability limits when compared with those provided by the IHC compensator for the same controller parameters (convergence and leakage gains). It should be noted that similar cascade solutions of band-pass filters have previously been developed in the context of periodic active noise control (ANC), for eliminating uncorrelated noise components appearing in the residual error signal and then amplified by the ANC system due to the effect of the secondary path (Kuo and Minjiang, 1996; Xu and Kuo, 2007).

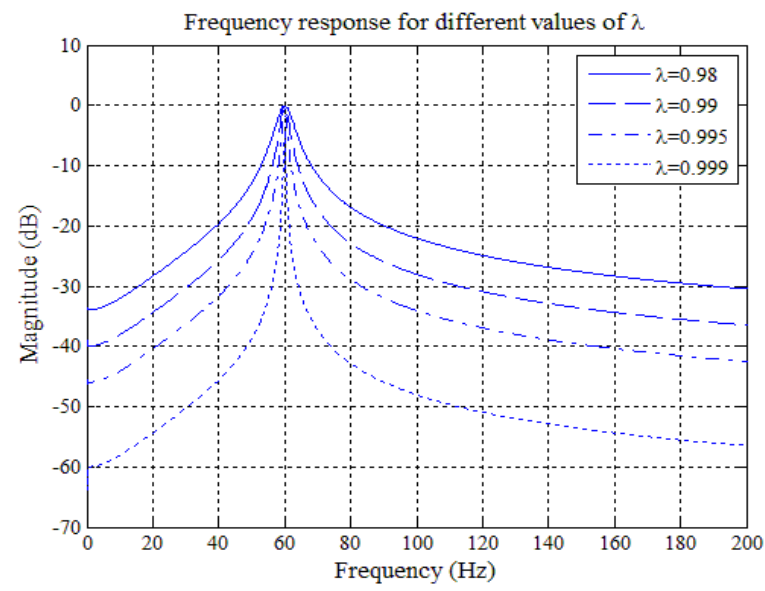

Figure 3: Frequency response of the RLS-fixed filter for different values of $\lambda$ (harmonic frequency $\left.\omega_{o}=60 \mathrm{~Hz}\right)$.

\section{Closed-Loop Pole Locations and Time Constants}

It is well known that for an LTI system the transient response is dictated by the location of the dominant closed-loop poles. In this section it will be shown that when the plant dynamics are assumed to be far from the compensators poles (Sievers and Von Flotow, 1992), the condition for closed-loop stability and response time for both compensators considered are very similar. This is also confirmed through simulations and experimental results presented in the next sections. 


\subsection{Closed loop pole locations and time constant for the IHC compensator.}

The determination of the location of the dominant closed-loop poles is based on the study undertaken in (Sievers and Von Flotow, 1992). The analysis assumes that the plant's dynamics are far from the compensator poles and that the poles of the loop transfer function matrix $K(z) G_{c}(z)$ are not repeated. The loop transfer function can then be represented as partial fractions using the residue of each pole. The number of partial fractions depends on the number of compensator and plant poles. In the disturbance frequency of interest, the loop transfer function is dominated by the influence of the pole at that frequency and therefore can be approximated with the partial fraction corresponding solely to that pole. The location of the closed loop poles for different values of the convergence and/or leakage gain can then be determined by the loop transfer function $K(z) G_{c}(z)$ in this frequency region using a root-locus argument (Maciejowski, 1989). For the IHC algorithm the equivalent LTI representation given by Eq.(4) can be rewritten in the following way:

$$
K_{I H C}(z)=-\beta A\left[\frac{\left(e^{j \omega_{o} T} e^{j \varphi}+e^{-j \omega_{0} T} e^{-j \varphi}\right) z-\alpha\left(e^{j \varphi}+e^{-j \varphi}\right)}{\left(z-\alpha e^{j \omega_{o} T}\right) \cdot\left(z-\alpha e^{-j \omega_{0} T}\right)}\right]
$$

The poles of the open-loop compensator are at $z_{1}=\alpha e^{j \omega_{0} T}$ and $z_{2}=\alpha e^{-j \omega_{0} T}$. Assuming that the open-loop transfer function $K(z) G_{c}(z)$ has a relative degree of at least one, the residue of the first pole of the loop transfer function can be described by:

$$
\operatorname{Residue}\left[K_{I H C}(z) G_{c}(z)\right]=\left.K_{I H C}(z) G_{c}(z) \cdot\left(z_{1}-\alpha e^{j \omega_{0} T}\right)\right|_{z_{1}=\alpha e^{j \omega_{0} T}}
$$

Therefore:

$$
\begin{gathered}
R_{I H C 1}=\left.\left[-\beta A\left[\frac{\left(e^{j \omega_{o} T} e^{j \varphi}+e^{-j \omega_{o} T} e^{-j \varphi}\right) z-\alpha\left(e^{j \varphi}+e^{-j \varphi}\right)}{\left(z-\alpha e^{j \omega_{o} T}\right) \cdot\left(z-\alpha e^{-j \omega_{o} T}\right)}\right] \cdot G_{c}\left(z_{1}\right) \cdot\left(z_{1}-\alpha e^{j \omega_{o} T}\right)\right]\right|_{z_{1}=\alpha e^{j \omega_{0} T}} \Rightarrow \\
R_{I H C 1}=-\beta C G_{c}\left(\alpha e^{j \omega_{o} T}\right) e^{j \omega_{o} T}
\end{gathered}
$$

In the same way, the residue $R_{2}$ for $z=\alpha e^{-j \omega_{0} T}$ can be found to be:

$$
R_{I H C 2}=-\beta C^{*} G_{c}\left(\alpha e^{-j \omega_{o} T}\right) e^{-j \omega_{o} T}
$$

Now, since it has been assumed that the poles of the loop transfer function $K_{I H C}(z) G_{c}(z)$ are not repeated, this can be represented in terms of its residues $R_{n}$ in the following way:

$$
K_{I H C}(z) G_{c}(z)=\frac{R_{I H C 1}}{\left(z-\alpha e^{j \omega_{o} T}\right)}+\frac{R_{I H C 2}}{\left(z-\alpha e^{-j \omega_{o} T}\right)}+\sum_{n=1}^{N} \frac{R_{n}}{\left(z-z_{n}\right)}
$$

The poles of the plant are at $z_{n}$ and for the compensator design are assumed, to be far from the compensator poles (Sievers and Von Flotow, 1992) or well damped. The loop transfer function can be rewritten as follows: 


$$
K_{I H C}(z) G_{c}(z)=\frac{-\beta \cdot C G_{c}\left(\alpha e^{j \omega_{o} T}\right) e^{j \omega_{o} T}}{\left(z-\alpha e^{j \omega_{o} T}\right)}+\frac{-\beta \cdot C^{*} G_{c}\left(\alpha e^{-j \omega_{o} T}\right) e^{-j \omega_{o} T}}{\left(z-\alpha e^{-j \omega_{o} T}\right)}+\sum_{n=1}^{N} \frac{R_{n}}{\left(z-z_{n}\right)}
$$

Observing Eq.(12) it is clear that, the transfer function, in the frequency band around $z=\alpha e^{j \omega_{o} T}$ is dominated by the first term. Hence the loop transfer function can be approximated by:

$$
K_{I H C}(z) G_{c}(z) \approx \frac{-\beta \cdot C \cdot G_{c}\left(\alpha e^{j \omega_{o} T}\right) e^{j \omega_{o} T}}{\left(z-\alpha e^{j \omega_{o} T}\right)}
$$

The closed-loop pole location for the system can be found using the root-locus argument of Eq.(14) (Maciejowski, 1989). If the $i^{\text {th }}$ pole $z_{i}$ is on the root-locus, then, it must satisfy the root-locus equation:

$$
K_{I H C}(z) G_{c}(z)=1
$$

The location of the $i^{\text {th }}$ closed-loop pole near $\alpha e^{j \omega_{o} T}$ can then be determined by solving the following equation in terms of $z_{I H C i}$ (Sievers and Von Flotow, 1992):

$$
\frac{-\beta C G_{c}\left(\alpha e^{j \omega_{o} T}\right) e^{j \omega_{o} T}}{\left(z_{I H C i}-\alpha e^{j \omega_{0} T}\right)}=1
$$

therefore

$$
z_{I H C i}=\alpha e^{j \omega_{o} T}-\beta C G_{c}\left(\alpha e^{j \omega_{o} T}\right) e^{j \omega_{o} T}
$$

Defining the term $M=C \times G_{c}\left(\alpha e^{j \omega_{o} T}\right)$, Eq.(16) becomes:

$$
z_{I H C i}=|\alpha-\beta \cdot M| e^{j \omega_{o}\left(T+\phi_{1}\right)}
$$

where $\phi_{1}=\tan ^{-1}\left(\frac{\operatorname{Im}(\alpha-\beta \cdot M)}{\operatorname{Re}(\alpha-\beta \cdot M)}\right)$ is the phase shift introduced by the complex number $(\alpha-\beta \cdot M)$. From Eq.(17) it is clear that, under the plant assumptions described above, the closed-loop system will be stable if the magnitude of each of the closed-loop poles at $\omega_{o}$ is less than unity

$$
|\alpha-\beta \cdot M|<1
$$

Also the system's decay time constant (Mulligan, 1949; Sievers and Von Flotow, 1992) is given by:

$$
\tau_{I H C} \approx \frac{T}{1-r_{e^{j \omega_{o} T}}} \approx \frac{T}{1-|\alpha-\beta \cdot M|}
$$

where $r_{e^{j \omega_{o} T}}$ is the magnitude of the closed-loop pole at the specified frequency. 
For $\alpha \approx 1$ or $C=G_{c}\left(\alpha e^{j \omega_{o} T}\right)^{-1}$ it can be assumed that $\mathrm{M}=C G_{c}\left(\alpha e^{j \omega_{o} T}\right) \approx 1$ and Eqs.(17), (19) respectively become:

$$
\begin{gathered}
z_{I H C i} \approx|\alpha-\beta| e^{j \omega_{o} T} \\
\tau_{I H C} \approx \frac{T}{1-|\alpha-\beta|}
\end{gathered}
$$

Observing Eq.(21) it is clear that as $\beta$ increases, the absolute value term of the denominator decreases, thereby decreasing the response time. In addition, the effect of the leakage term $\alpha$ in the system's response can also be seen. As $\alpha$ decreases the response time is also decreasing. That is; the decay time constant of the algorithm is inversely proportional to the convergence gain $\beta$ and proportional to the leakage gain $\alpha$.

\subsection{Closed loop pole locations and time constant for the RLS based IHC compensator.}

For the RLS based IHC algorithm the equivalent LTI representation given by Eq.(5) can be written as:

$$
K_{R L S}(z)=-\frac{4(1-\lambda) \beta A \cdot\left[\left[z \cos \left(\omega_{o} T+\varphi\right)-\alpha \cos (\varphi)\right] \cdot\left[z^{2}-\cos \left(\omega_{o} T\right) z\right]\right]}{\left(z-z_{1}\right) \cdot\left(z-z_{2}\right) \cdot\left(z-z_{3}\right) \cdot\left(z-z_{4}\right)}
$$

with

$$
\begin{gathered}
z_{1}=\alpha e^{j \omega_{o} T} \\
z_{2}=\alpha e^{-j \omega_{o} T} \\
z_{3,4}=\frac{\cos \left(\omega_{o} T\right) \pm \sqrt{\cos ^{2}\left(\omega_{o} T\right)-\left(2 \lambda-\lambda^{2}\right)}}{(2-\lambda)}
\end{gathered}
$$

Using the trigonometric property $\cos (\theta)=\frac{e^{j \theta}+e^{-j \theta}}{2}$ for $\theta_{1}=\omega_{o} T+\varphi, \theta_{2}=\varphi$ and $\theta_{3}=\omega_{o} T$ the loop transfer function matrix becomes:

$K_{R L S}(z) G_{c}(z)=-(1-\lambda) \beta A\left[\frac{\left[\left[\left(e^{j \omega_{o} T} e^{j \varphi}+e^{-j \omega_{o} T} e^{-j \varphi}\right) z-\alpha\left(e^{j \varphi}+e^{-j \varphi}\right)\right] \cdot\left[2 z^{2}-\left(e^{j \omega_{o} T}+e^{-j \omega_{o} T}\right) z\right]\right]}{\left(z-z_{1}\right)\left(z-z_{2}\right)\left(z-z_{3}\right)\left(z-z_{4}\right)}\right] G_{c}(z)(23)$

Observing the poles of the compensator leads to the conclusion that two cases could be considered. First, when $\alpha \neq \lambda$ the compensator has four different poles and the analysis will be as for the IHC algorithm. The second case occurs when $\alpha=\lambda$ and they are both less than unity. Then the compensator would have two repeated poles and the residue of each pole should be calculated in a different way, leading to a different solution. Here the analysis and simulated results are presented only for the first case $(\alpha \neq \lambda)$, since the second case is unlikely to occur in practice. It is argued here that in real applications, in order to provide suitable adaption capability for the RLS algorithm, the value of the forgetting factor $\lambda$ should not exceed a value of 0.99 . If the leakage gain $\alpha$ takes this value or lower, the vibration attenuation that could be achieved would be insignificant. In real applications the minimum value of $\alpha$ is 
usually much closer to unity which adds a degree of robustness to the system, albeit with some loss relative to the maximum achievable vibration attenuation (Zazas, 2009). Since the first case is considered and the poles of the loop transfer function matrix are not repeated, the latter can be written in terms of its residues $R_{n}$ in the following way:

$$
K_{R L S}(z) G_{c}(z)=\frac{R_{R L S 1}}{\left(z-z_{1}\right)}+\frac{R_{R L S 2}}{\left(z-z_{2}\right)}+\frac{R_{R L S 3}}{\left(z-z_{3}\right)}+\frac{R_{R L S 4}}{\left(z-z_{4}\right)}+\sum_{n=1}^{N} \frac{R_{n}}{\left(z-z_{n}\right)}
$$

As for the IHC algorithm to obtain $R_{R L S 1}$ multiply $K_{R L S}(z) G_{c}(z)$ with $\left(z-z_{1}\right)$ and evaluate for $z=z_{1}$ to give:

$$
R_{R L S 1}=-\frac{\beta \alpha(1-\lambda) \cdot\left[(2 \alpha-1) e^{2 j \omega_{o} T}-1\right]}{\left(z_{1}-z_{3}\right) \cdot\left(z_{1}-z_{4}\right) e^{-j \omega_{o} T}} \cdot C G_{c}\left(\alpha e^{j \omega_{o} T}\right)
$$

Working in the same way for $R_{2}, R_{3}$ and $R_{4}$ leads to:

$$
\begin{gathered}
R_{R L S 2}=-\frac{\beta \alpha(1-\lambda) \cdot\left[(2 \alpha-1) e^{-2 j \omega_{o} T}-1\right]}{\left(z_{2}-z_{3}\right) \cdot\left(z_{2}-z_{4}\right) e^{j \omega_{o} T}} \cdot C^{*} G_{c}\left(\alpha e^{-j \omega_{o} T}\right) \\
R_{R L S 3}=-(1-\lambda) \beta A\left[\frac{\left[\left(e^{j \omega_{o} T} e^{j \varphi}+e^{-j \omega_{o} T} e^{-j \varphi}\right) z_{3}-\alpha\left(e^{j \varphi}+e^{-j \varphi}\right)\right] \cdot\left[2 z_{3}^{2}-z_{3} e^{j \omega_{o} T}-z_{3} e^{-j \omega_{o} T}\right]}{\left(z_{3}-z_{1}\right) \cdot\left(z_{3}-z_{2}\right) \cdot\left(z_{3}-z_{4}\right)}\right] G_{c}\left(z_{3}\right) \\
R_{R L S 4}=-(1-\lambda) \beta A\left[\frac{\left[\left[\left(e^{j \omega_{o} T} e^{j \varphi}+e^{-j \omega_{o} T} e^{-j \varphi}\right) z_{4}-\alpha\left(e^{j \varphi}+e^{-j \varphi}\right)\right] \cdot\left[2 z_{4}^{2}-z_{4} e^{j \omega_{o} T}-z_{4} e^{-j \omega_{o} T}\right]\right]}{\left(z_{4}-z_{1}\right) \cdot\left(z_{4}-z_{2}\right) \cdot\left(z_{4}-z_{3}\right)}\right] G_{c}\left(z_{4}\right)(28)
\end{gathered}
$$

In the frequency band around $z=\alpha e^{j \omega_{0} T}$ and when $\alpha>\lambda$, the transfer function matrix is dominated by the first term (given the above assumption about the plant dynamics). Hence the loop transfer function becomes:

$$
K_{R L S}(z) G_{c}(z) \approx \frac{R_{R L S 1}}{\left(z-z_{1}\right)}
$$

If the $i t h$ pole $z_{i}$ is on the root-locus then, as above, it must satisfy the root-locus equation:

$$
K_{R L S}(z) G_{c}(z)=1
$$

The location of the ith closed-loop pole near $\alpha e^{j \omega_{o} T}$ can be determined by solving the following equation in terms of $z_{i}$ :

$$
\frac{R_{R L S i}}{\left(z_{R L S i}-z_{i}\right)}=1
$$


This becomes:

$$
z_{R L S 1}=z_{1}+R_{R L S 1}
$$

or using Eq.(25)

$$
z_{R L S i} \approx|\alpha-\underbrace{\frac{\alpha(1-\lambda) \cdot\left[(2 \alpha-1) e^{2 j \omega_{o} T}-1\right]}{\left(z_{1}-z_{3}\right) \cdot\left(z_{1}-z_{4}\right)}}_{L} \cdot \beta \cdot M| e^{j \omega_{o}\left(T+\phi_{2}\right)}
$$

where once again $M=C \cdot G_{c}\left(\alpha e^{j \omega_{o} T}\right)$ and $\phi_{2}=\tan ^{-1}\left(\frac{\operatorname{Im}(\alpha-L \cdot \beta \cdot M)}{\operatorname{Re}(\alpha-L \cdot \beta \cdot M)}\right)$. Then as before the systems decay time constant is given by:

$$
\tau_{R L S} \approx \frac{T}{1-|\alpha-\underbrace{\frac{\alpha(1-\lambda) \cdot\left[(2 \alpha-1) e^{2 j \omega_{o} T}-1\right]}{\left(z_{1}-z_{3}\right) \cdot\left(z_{1}-z_{4}\right)}}_{L} \cdot \beta \cdot M|}
$$

Now as for the IHC, for $\alpha \approx 1$ or $C=G_{c}\left(\alpha e^{j \omega_{o} T}\right)^{-1}$, it can be assumed that $M=C \cdot G_{c}\left(\alpha e^{j \omega_{o} T}\right)=1$ and Eqs.(33) and (34) become:

$$
\begin{gathered}
z_{R L S i} \approx|\alpha-L \cdot \beta| e^{j \omega_{o} T} \\
\tau_{R L S} \approx \frac{T}{1-|\alpha-L \cdot \beta|}
\end{gathered}
$$

Observing Eq.(36), the term $L$ is clearly complex and the influence of $\alpha, \beta$ and $\lambda$ to the algorithm's speed of convergence is not straight-forward to determine. It can be shown, however, that for $\alpha$ close to unity (i.e. $\alpha \cong 1$ ), the real and imaginary part of $L$ respectively become $\operatorname{Re}\{L\}=2-\lambda$ and $\operatorname{Im}\{L\}=0$ (see Appendices 2, 3). Then Eqs.(20), (21), (35) and (36) can further be simplified as will be shown in the next section.

\section{Stability and Convergence Analysis}

A schematic diagram of the experimental test rig used to validate the theoretical findings is shown in Fig. 4. A meter long square solid metal beam with cross section area of $100 \mathrm{~cm}^{2}$ is mounted on a flexible bench using two elastomeric mounts. A $170 \mathrm{~N}$ Gearing \& Watson inertial actuator is attached to the middle point of the beam. This is used to excite the system and represents the disturbance forces that may occur in practice. In addition, two 10N LabWorks FG-142A inertial actuators are attached at both ends of the beams to provide the appropriate control forces. The control objective is to minimise the acceleration transmitted to the flexible foundation using acceleration measurements captured at the same locations as 
the attached control actuators. A typical control application described by such configuration is the minimisation of the acceleration transmitted to the foundation, caused by heavy machinery sited on the top of a raft structure which is itself resiliently mounted to the foundation. At its most complex the system has two inputs (control forces) and two outputs (axial acceleration measurements). By adding the axial acceleration measurements and feeding the same control signal to both control actuators the system is transformed to a Single Input-Single Output (SISO) and since the theoretical findings are based on the assumption of a SISO system, this experimental configuration was used.

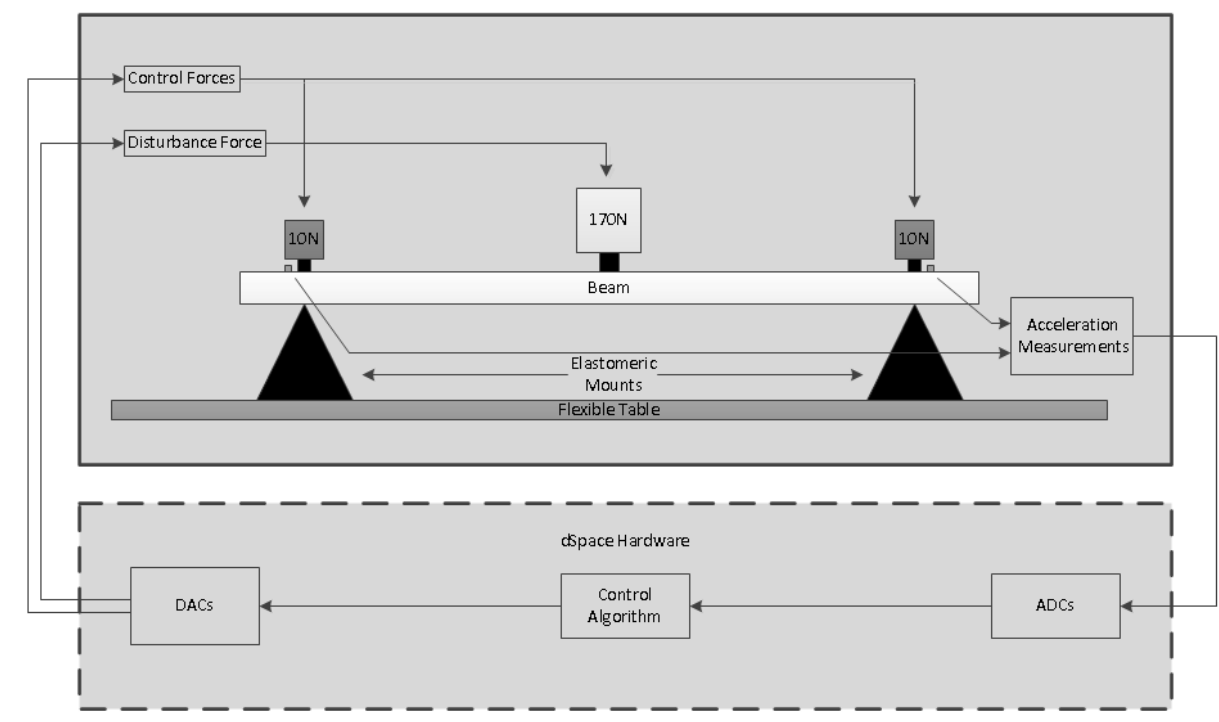

Figure 4: Schematic diagram of the experimental test-rig.

The magnitude of the frequency response of the Disturbance Path (disturbance actuator to sum of axial acceleration) is shown in Fig. 5 (left plot). It is clear that the system has a resonant peak at $284 \mathrm{~Hz}$ (corresponding to the first bending mode). Also shown in the same figure (right plot) is the magnitude of the frequency response of the Control Path (control actuators to sum of axial acceleration). The control shakers also excite the first bending mode and the $284 \mathrm{~Hz}$ resonance is again evident. The transfer function coefficients of the disturbance path and control path models used for the simulation presented herein where identified from experimentally derived frequency response functions and are listed in Appendix 4.
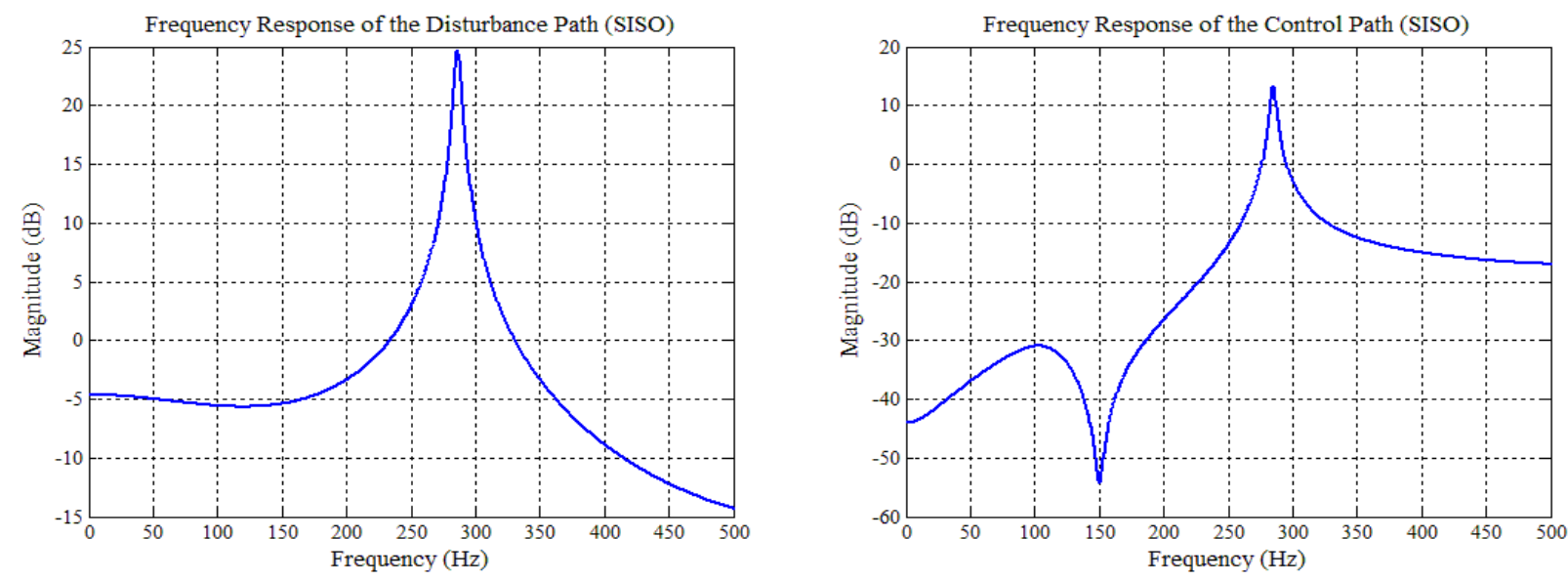

Figure 5: Magnitude of frequency responses - Left) Disturbance actuator to Sum acceleration, Right) Control actuators to Sum acceleration. 
Since both algorithms have an equivalent LTI feedback representation, any method from the LTI framework can be used to analyse the stability of the closed-loop system. The methods used for this exercise were the Nyquist criterion, the open-loop frequency responses and the determination of the closed-loop dominant poles. The control path model $G_{c}$ used for stability analysis and simulations is a reduced order transfer function model obtained from frequency response measurements and describes the dynamics of the actuators, amplifiers and filters used. This was done in order to observe the closed-loop pole locations at the frequencies of interest more accurately without invalidating the general behaviour of the algorithms. The operator $C$ used to both algorithms is the inverse of the control path model evaluated at the disturbance frequency and is a complex scalar since the SISO case is considered.

The values of the forgetting factor $\lambda$ and leakage gain $\alpha$ are set to 0.98 and 0.999998 respectively in order to combine good steady state performance with adaptability. For $\alpha=0.999998 \cong 1$ Eqs. (20), (21), (35) and (36) become:

$$
\begin{gathered}
z_{I H C i} \approx|1-\beta| e^{j \omega_{o} T} \\
\tau_{I H C} \approx \frac{T}{\beta} \\
z_{R L S i} \approx|1-(2-\lambda) \beta| e^{j \omega_{o} T} \\
\tau_{R L S} \approx \frac{T}{(2-\lambda) \beta}
\end{gathered}
$$
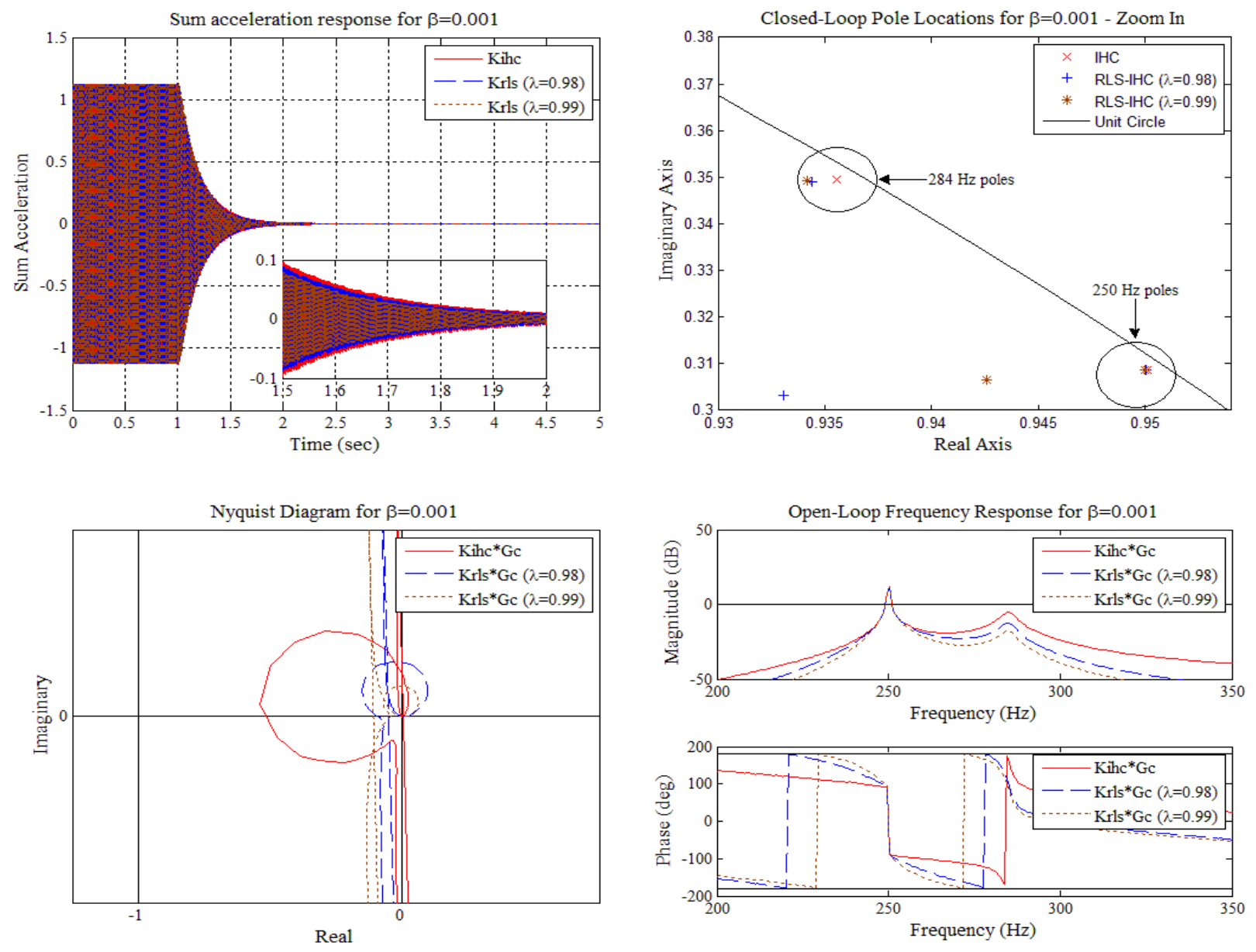

Figure 6: Time series during convergence, closed-loop pole locations, Nyquist diagram and Bode plots for $\beta=0.001$. 
From the above equations it is clear that, at the disturbance frequency, the influence of the forgetting factor (for commonly used values) on the stability and speed of convergence for any value of the convergence gain is negligible. To better illustrate how the forgetting factor affects the system's closed-loop stability (and as a consequence speed of convergence) the case when the disturbance signal is a sinusoid with frequency at $250 \mathrm{~Hz}$ is considered (close to the resonance peak). Figs. 6 to 8 shows, the simulated time series of the system's output during convergence (sum acceleration response), the locations of the closed-loop system's poles, the Nyquist diagram and the open loop frequency response of systems for three different values of the convergence gain $\beta$. For the RLS-IHC algorithm the case when the forgetting factor has a value of 0.99 is also considered. It should be noted that the time series responses of the system's outputs were obtained through simulations using the frequency domain update implementations to examine the accuracy of the fixed parameter compensators representations when performing stability analysis.
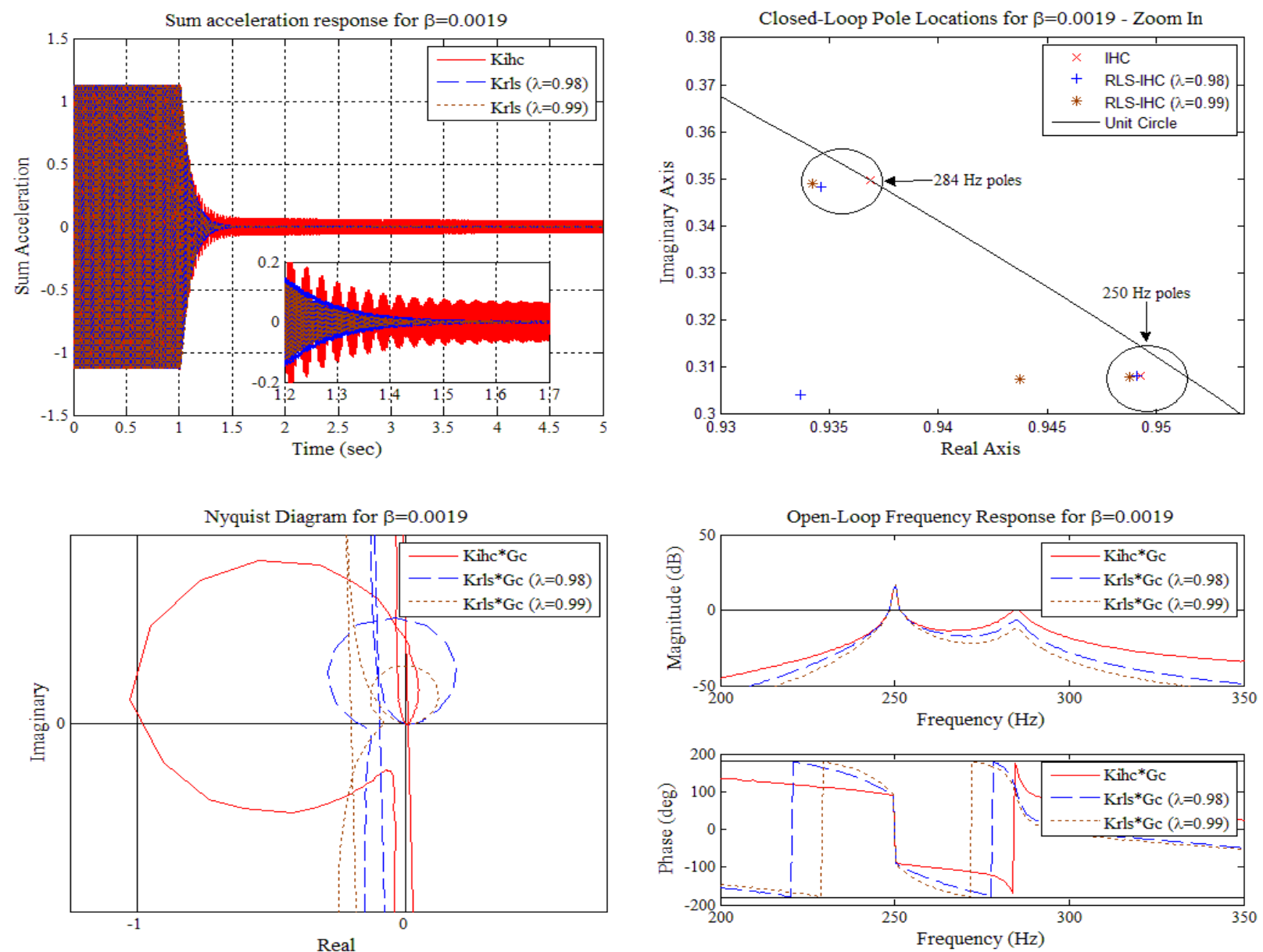

Figure 7: Time series during convergence, closed-loop pole locations, Nyquist diagram and Bode plots for $\beta=0.0019$.

When the closed-loop system for all three cases is stable $(\beta=0.001)$, the convergence speeds for both implementations are almost identical (responses overlap in the time series plot of Fig. 6). This was expected since the locations of the dominant closed loop poles (at $250 \mathrm{~Hz}$ ) for all three cases are very close to each other as indicated from the pole locations plot of Fig. 6 and as predicted by the analysis in Section 4. More importantly, the effect of the additional filtering action introduced by the RLS fixed filter (for the RLS-IHC algorithm) on the system's closed-loop stability can be seen in both Nyquist plot and the open-loop frequency responses of Fig. 6. Better stability margins are achieved as the side-bands of the 
open-loop frequency response are less amplified (Kuo and Minjiang, 1996). This leads to the conclusion that the narrower the bandwidth of the RLS fixed filter (which is controlled by the forgetting factor $\lambda$ ), the less amplified the side-bands of the open-loop frequency response will be. As a result, close by resonances will be less amplified when the RLS-IHC is used, thereby providing better stability limits when compared with those obtained by the IHC algorithm for the same controller parameters (convergence and leakage gain).

When the value of the convergence gain is increased to $\beta=0.0019$ all plots of Fig. 7 indicate that for the IHC implementation the closed-loop system is critically stable. The system's speed of convergence no longer depends on the location of the $250 \mathrm{~Hz}$ poles, but to those associated with the resonance peak at $284 \mathrm{~Hz}$, as now these are the dominant ones (closer to the circumference of the unit circle). For the same value of the convergence gain and for both RLS based implementations, the closedloop systems as expected remain stable. Finally by further increasing the convergence gain to $\beta=0.002$, the closed loop system turns unstable when the IHC algorithm is used (at the resonance frequency of 284 $\mathrm{Hz}$ ) as indicated from all plots of Fig. 8. In contrast, when the RLS based algorithm is used the closed loop system still remains stable. As a result, the convergence gain can further be increased to accelerate convergence to the optimum solution.
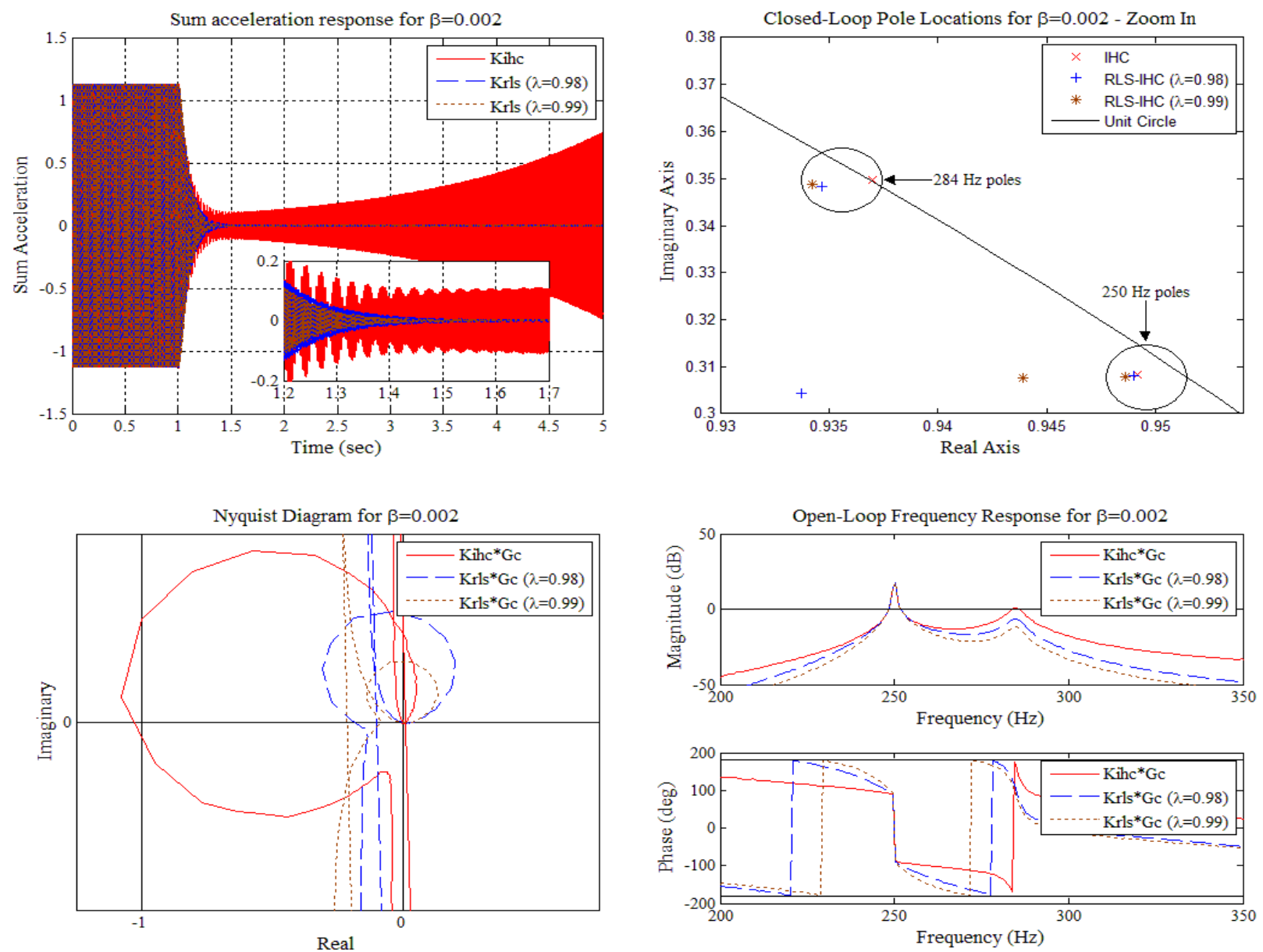

Figure 8: Time series during convergence, closed-loop pole locations, Nyquist diagram and Bode plots for $\beta=0.002$. 


\section{Experimental Validation}

The performance of both frequency domain algorithms were tested experimentally using the system described in the previous section (Fig. 4). Fig. 9 shows the sum acceleration response during convergence and the corresponding Power Spectral Density (PSD) plots for $\beta=0.002$ and for both implementations. From the PSD plot, it is clear that both algorithms provide the same steady state vibration attenuation at the disturbance frequency. For the IHC implementation though, the closed-loop system is critically stable since the $284 \mathrm{~Hz}$ resonance is slightly excited. In addition, the time series response indicates that both algorithms converge to the optimum solution at the same rate as expected. For the IHC implementation though this is the fastest rate that can be achieved, as a further increment of the convergence gain results in an unstable closed-loop system.
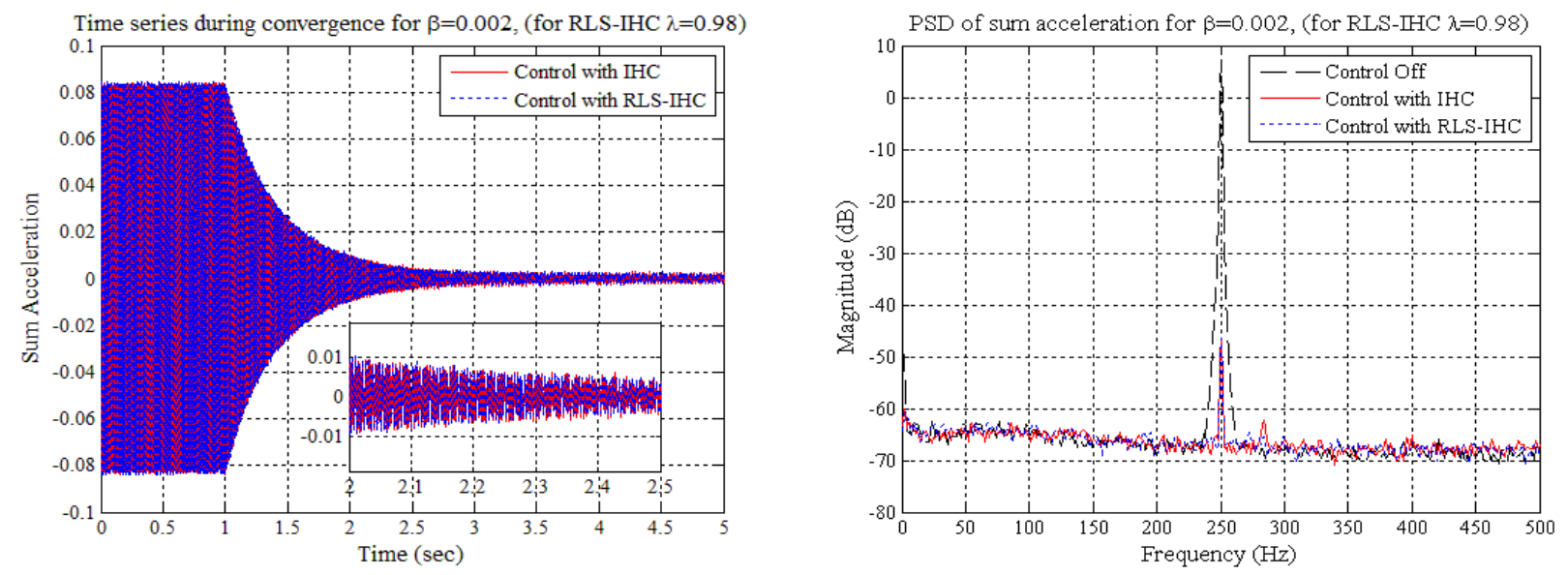

Figure 9: Time series during convergence and PSD of the sum acceleration for $\beta=0.002$.
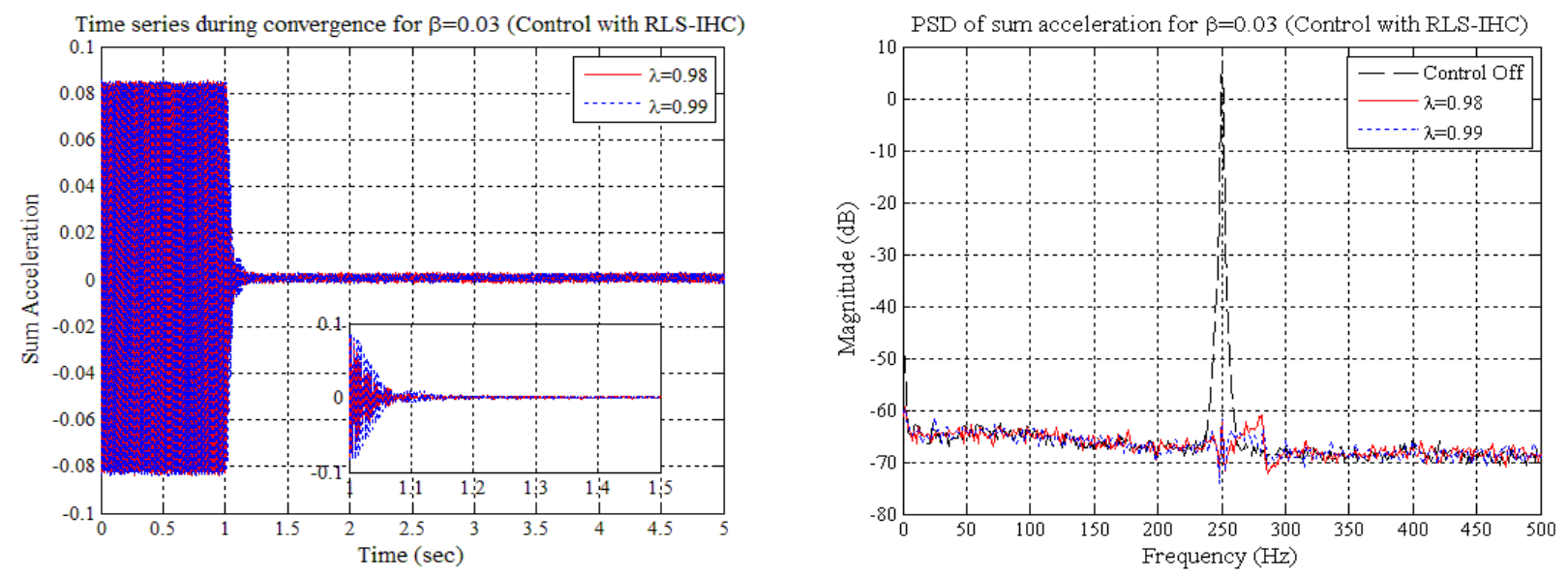

Figure 10: Time series during convergence and PSD of the sum acceleration for $\beta=0.03$.

For the RLS-IHC implementation the $284 \mathrm{~Hz}$ resonance peak starts to become problematic when the convergence gain is increased to $\beta=0.03$ as shown from the PSD plot of Fig. 10. Also shown in the same plot the PSD for the same value of $\beta$ but with the forgetting factor changed to $\lambda=0.99$. It is clear that this increment, improves the stability margin and results in less amplification of the resonance peak without any loss of convergence speed as expected. In addition due to the convergence gain increment, additional steady-state vibration attenuation is achieved at the disturbance frequency. Finally, observing 
the time series plot during convergence it is obvious that the system's output converges to the noise floor much faster when compared with the IHC implementation.

\section{Conclusions}

The LTI representations of two recently developed HC algorithms were utilised to analyse their convergence behaviour and stability robustness in a vibration control application. Mathematical derivations of the locations of the dominant closed-loop poles have explained the previously observed similarity in transient responses of both algorithms for small values of the convergence gain or when the disturbance frequency is far from a resonant peak. In addition, it was shown that the fact that the RLSIHC algorithm can be derived as a cascade combination of two individual band-pass filters both centered on the disturbance frequency, explains the better stability margins provided by the algorithm when compared with those provided by the IHC algorithm for the same values of the convergence and leakage gains. This is especially true for modally dense systems where resonant peaks in near side-band regions are more likely to influence the system stability for the IHC algorithm. As a result, the value of the convergence gain can further increased for the RLS-IHC algorithm so the system can reach the optimum solution faster. For these reasons, the RLS-IHC algorithm is more likely to be the preferred choice for applications where the controller is required to converge to the optimum solution as rapidly as possible and for all variations (i.e. control of noise and $\backslash$ or vibration produced by variable speed engines or motors). 


\section{Appendix 1: Nomenclature}

\begin{tabular}{|c|c|}
\hline$q^{-1}$ & Backward shift operator \\
\hline$\omega_{o}$ & Disturbance frequency \\
\hline$G_{c}(q)$ & Control path model \\
\hline$G_{c}\left(e^{j \omega_{o} T}\right)$ & Control path frequency response evaluated at disturbance frequency \\
\hline$G_{c}(z)$ & Control path transfer function in $\mathrm{Z}$ domain \\
\hline$G_{c}\left(e^{j \omega_{o} T}\right)^{H}$ & $\begin{array}{l}\text { Hermitian transpose of the control path frequency response evaluated at the disturbance } \\
\text { frequency }\end{array}$ \\
\hline$G_{d}(q)$ & Disturbance path model \\
\hline$G_{d}\left(e^{j \omega_{o} T}\right)$ & Disturbance path frequency response evaluated at disturbance frequency \\
\hline C & Function of $G_{c}\left(e^{j \omega_{o} T}\right)$ used in gradient descent algorithm \\
\hline$A$ & Amplitude of complex number $C$ for SISO case \\
\hline$\phi$ & Phase of complex number $C$ for SISO case \\
\hline$P$ & Amplitude of disturbance sinusoid \\
\hline$t$ & Time in seconds \\
\hline$\varphi$ & Phase of disturbance sinusoid \\
\hline$d_{t}$ & Disturbance sinusoid in time domain \\
\hline$e_{t}$ & Error signal in time domain \\
\hline$u_{t}$ & Control signal in time domain \\
\hline$z_{t}$ & Reference signal in time domain \\
\hline$u\left(e^{j \omega_{o} T}\right)$ & Control signal in frequency domain \\
\hline$e\left(e^{j \omega_{o} T}\right)$ & Error signal in frequency domain \\
\hline$\hat{e}\left(e^{j \omega_{o} T}\right)$ & Estimate of the error signal in frequency domain \\
\hline$T$ & Sampling period \\
\hline $\begin{array}{l}k \\
\alpha\end{array}$ & $\begin{array}{l}\text { Iteration index } \\
\text { Leakage gain }\end{array}$ \\
\hline$\beta$ & Convergence gain \\
\hline$K_{I H C}(z)$ & Transfer function representation of the IHC algorithm in $Z$ domain \\
\hline$K_{R L S}(z)$ & $\begin{array}{l}\text { Transfer function representation of the Recursive Least Squares based IHC algorithm in } Z \\
\text { domain }\end{array}$ \\
\hline$G_{R L S}(z)$ & Transfer function representation of the Recursive Least Squares algorithm in $Z$ domain \\
\hline$\lambda$ & Forgetting factor \\
\hline$z_{i}$ & $i^{t h}$ closed loop system pole \\
\hline$R_{i}$ & $i^{\text {th }}$ pole residue \\
\hline$R_{n}$ & Pole residues of the loop transfer function \\
\hline$\tau$ & Decay time constant \\
\hline$r_{e^{j \omega_{0} T}}$ & Magnitude of closed loop pole evaluated at the disturbance frequency \\
\hline
\end{tabular}


Appendix 2: Proof of the following condition:

$$
\text { If } \alpha \cong 1 \text { then } \operatorname{Im}\{L\} \cong 0
$$

First expand $L$ to take the form $L=\operatorname{Re}\{L\}+\operatorname{Im}\{L\} j$.

$$
L=\frac{\alpha(1-\lambda)\left((2 \alpha-1) e^{2 j \omega_{o} T}-1\right)}{\left(z_{1}-z_{3}\right)\left(z_{1}-z_{4}\right)}=\frac{\operatorname{Num}(L)}{\operatorname{Den}(L)}
$$

with

$$
\begin{gathered}
z_{1}=\alpha e^{j \omega_{o} T} \\
z_{3,4}=\frac{\cos \left(\omega_{o} T\right) \pm \sqrt{\cos ^{2}\left(\omega_{o} T\right)-\left(2 \lambda-\lambda^{2}\right)}}{2-\lambda}
\end{gathered}
$$

The numerator of $L$ becomes:

$$
\begin{gathered}
\operatorname{Num}(L)=\alpha(1-\lambda)\left((2 \alpha-1) e^{2 j \omega_{o} T}-1\right) \\
=\alpha(1-\lambda)\left((2 \alpha-1)\left(\cos \left(2 \omega_{o} T\right)+\sin \left(2 \omega_{o} T\right) j\right)-1\right) \Rightarrow \\
\operatorname{Num}(L)=\underbrace{\alpha(1-\lambda)\left((2 \alpha-1) \cos \left(2 \omega_{o} T\right)-1\right)}_{\operatorname{Re}\{\operatorname{Num}(L)\}=a}+\underbrace{\alpha(1-\lambda)(2 \alpha-1) \sin \left(2 \omega_{o} T\right)}_{\operatorname{Im}\{\operatorname{Num}(L)\}=b} j
\end{gathered}
$$

In the same way the denominator of $L$ becomes:

$$
\operatorname{Den}(L)=\left(z_{1}-z_{3}\right)\left(z_{1}-z_{4}\right)=z_{1}^{2}-z_{1}\left(z_{3}+z_{4}\right)+z_{3} z_{4}
$$

Working each term individually we get:

$$
\begin{gathered}
z_{1}^{2}=\alpha^{2} e^{2 j \omega_{o} T}=\alpha^{2} \cos \left(2 \omega_{o} T\right)+\alpha^{2} \sin \left(2 \omega_{o} T\right) j \\
z_{3}+z_{4}=\frac{2 \cos \left(\omega_{o} T\right)}{2-\lambda} \Rightarrow-z_{1}\left(z_{3}+z_{4}\right)=-\frac{2 \alpha \cos ^{2}\left(\omega_{o} T\right)}{2-\lambda}-\frac{2 \alpha \sin \left(\omega_{o} T\right) \cos \left(\omega_{o} T\right)}{2-\lambda} j \\
z_{3} z_{4}=\frac{\cos ^{2}\left(\omega_{o} T\right)-\cos ^{2}\left(\omega_{o} T\right)+\left(2 \lambda-\lambda^{2}\right)}{(2-\lambda)^{2}}=\frac{\lambda}{2-\lambda}
\end{gathered}
$$

Using the above equations $\operatorname{Den}(L)$ becomes:

$$
\operatorname{Den}(L)=\underbrace{\left(\frac{\alpha^{2}(2-\lambda) \cos \left(2 \omega_{o} T\right)-2 \alpha \cos ^{2}\left(\omega_{o} T\right)+\lambda}{2-\lambda}\right)}_{\operatorname{Re}\{\operatorname{Den}(L)\}=c}+\underbrace{\left(\frac{\alpha^{2}(2-\lambda) \sin \left(2 \omega_{o} T\right)-2 \alpha \sin \left(\omega_{o} T\right) \cos \left(\omega_{o} T\right)}{2-\lambda}\right) j(A)}_{\operatorname{Im}\{\operatorname{Den}(L)\}=d}
$$


Now, using Eqs. (A.3) and (A.4) the term $L$ gets the form:

$$
L=\frac{a+b j}{c+d j}=\frac{(a+b j)(c-d j)}{(c+d j)(c-d j)}=\frac{a c-a d j+b g j+b d}{c^{2}+d^{2}}=\frac{a c+b d}{\underbrace{c^{2}+d^{2}}_{\operatorname{Re}\{L\}}}+\underbrace{\frac{b c-a d}{c^{2}+d^{2}}}_{\operatorname{Im}\{L\}} j
$$

The imaginary part of $L$ is approaching zero if $b c \cong a d$ since $c^{2}+d^{2}$ is a finite number. Working the two terms ( $b c$ and $a d$ ) in parallel we get:

$$
\begin{aligned}
& b c=\alpha(1-\lambda)(2 \alpha-1) \sin \left(2 \omega_{o} T\right)\left(\frac{\alpha^{2}(2-\lambda) \cos \left(2 \omega_{o} T\right)-2 \alpha \cos ^{2}\left(\omega_{o} T\right)+\lambda}{2-\lambda}\right) \\
& \left.a d=\alpha(1-\lambda)\left((2 \alpha-1) \cos \left(2 \omega_{o} T\right)-1\right)\left(\frac{\alpha^{2}(2-\lambda) \sin \left(2 \omega_{o} T\right)-2 \alpha \sin \left(\omega_{o} T\right) \cos \left(\omega_{o} T\right)}{2-\lambda}\right)\right) \stackrel{b c \cong a d}{\Rightarrow} \\
& \left((2 \alpha-1) \sin \left(2 \omega_{o} T\right)\right)\left(\alpha^{2}(2-\lambda) \cos \left(2 \omega_{o} T\right)-2 \alpha \cos ^{2}\left(\omega_{o} T\right)+\lambda\right) \cong \\
& \left((2 \alpha-1) \cos \left(2 \omega_{o} T\right)-1\right)\left(\alpha^{2}(2-\lambda) \sin \left(2 \omega_{o} T\right)-2 \alpha \sin \left(\omega_{o} T\right) \cos \left(\omega_{o} T\right)\right) \Rightarrow \\
& \alpha^{2}(2 \alpha-1)(2-\lambda) \sin \left(2 \omega_{o} T\right) \cos \left(2 \omega_{o} T\right)-2 \alpha(2 \alpha-1) \sin \left(2 \omega_{o} T\right) \cos ^{2}\left(\omega_{o} T\right)+\lambda(2 \alpha-1) \sin \left(2 \omega_{o} T\right) \cong \\
& \alpha^{2}(2 \alpha-1)(2-\lambda) \sin \left(2 \omega_{o} T\right) \cos \left(2 \omega_{o} T\right)-2 \alpha(2 \alpha-1) \cos \left(2 \omega_{o} T\right) \sin \left(\omega_{o} T\right) \cos \left(\omega_{o} T\right) \\
& -\alpha^{2}(2-\lambda) \sin \left(2 \omega_{o} T\right)+2 \alpha \sin \left(\omega_{o} T\right) \cos \left(\omega_{o} T\right) \Rightarrow \\
& 2 \alpha(2 \alpha-1) \sin \left(2 \omega_{o} T\right) \cos ^{2}\left(\omega_{o} T\right)-\lambda(2 \alpha-1) \sin \left(2 \omega_{o} T\right) \cong \\
& 2 \alpha(2 \alpha-1) \cos \left(2 \omega_{o} T\right) \sin \left(\omega_{o} T\right) \cos \left(\omega_{o} T\right)+\alpha^{2}(2-\lambda) \sin \left(2 \omega_{o} T\right)-2 \alpha \sin \left(\omega_{o} T\right) \cos \left(\omega_{o} T\right)
\end{aligned}
$$

Using the following trigonometric identities

$\sin \left(2 \omega_{o} T\right)=2 \sin \left(\omega_{o} T\right) \cos \left(\omega_{o} T\right)$ and $\cos \left(2 \omega_{o} T\right)=\cos ^{2}\left(\omega_{o} T\right)-\sin ^{2}\left(\omega_{o} T\right)$ Eq. (A.6) becomes:

$$
\begin{aligned}
& \left(4 \alpha(2 \alpha-1) \cos ^{2}\left(\omega_{o} T\right)-2 \lambda(2 \alpha-1)\right) \sin \left(\omega_{o} T\right) \cos \left(\omega_{o} T\right) \cong \\
& \left(2 \alpha(2 \alpha-1) \cos \left(2 \omega_{o} T\right)+2 \alpha^{2}(2-\lambda)-2 \alpha\right) \sin \left(\omega_{o} T\right) \cos \left(\omega_{o} T\right) \Rightarrow \\
& 4 \alpha(2 \alpha-1) \cos ^{2}\left(\omega_{o} T\right)-2 \lambda(2 \alpha-1) \cong 2 \alpha(2 \alpha-1) \cos \left(2 \omega_{o} T\right)+2 \alpha^{2}(2-\lambda)-2 \alpha \Rightarrow \\
& 2 \alpha(2 \alpha-1)\left(\cos ^{2}\left(\omega_{o} T\right)+\sin ^{2}\left(\omega_{o} T\right)\right)-2 \lambda(2 \alpha-1) \cong 2 \alpha^{2}(2-\lambda)-2 \alpha \Rightarrow \\
& 2 \alpha^{2}-\alpha-\lambda(2 \alpha-1) \cong 2 \alpha^{2}-\alpha^{2} \lambda-\alpha \Rightarrow \\
& \alpha^{2}-2 \alpha+1 \cong 0 \Rightarrow(\alpha-1)^{2} \cong 0
\end{aligned}
$$

This is true if $\alpha \cong 1$ 
Appendix 3: Proof of the following condition:

$$
\text { If } \alpha \cong 1 \text { then } \operatorname{Re}\{L\}=2-\lambda
$$

For $\alpha \cong 1$ the numerator and denominator of $L$ become:

$$
\begin{aligned}
& \operatorname{Num}(L)=\underbrace{(1-\lambda)\left(\cos \left(2 \omega_{o} T\right)-1\right)}_{\operatorname{Re}\{\operatorname{Num}(L)\}=a}+\underbrace{(1-\lambda) \sin \left(2 \omega_{o} T\right)}_{I M\{\operatorname{Num}(L)\}=b} j \\
& \operatorname{Den}(L)=\underbrace{\left.\frac{(2-\lambda) \cos \left(2 \omega_{o} T\right)-2 \cos ^{2}\left(\omega_{o} T\right)+\lambda}{2-\lambda}\right)}_{\operatorname{Re}\{\operatorname{Den}(L)\}=c}+\underbrace{\left(\frac{(2-\lambda) \sin \left(2 \omega_{o} T\right)-2 \sin \left(\omega_{o} T\right) \cos \left(\omega_{o} T\right)}{2-\lambda}\right)}_{\operatorname{Im}\{\operatorname{Den}(L)\}=d} j
\end{aligned}
$$

As before $L=\underbrace{\frac{a c+b d}{c^{2}+d^{2}}}_{\operatorname{Re}\{L\}}+\underbrace{\frac{b c-a d}{c^{2}+d^{2}}}_{\operatorname{Im}\{L\}} j$

Using the following trigonometric identities

$$
\begin{gathered}
\sin \left(2 \omega_{o} T\right)=2 \sin \left(\omega_{o} T\right) \cos \left(\omega_{o} T\right) \\
\cos \left(2 \omega_{o} T\right)=2 \cos ^{2}\left(2 \omega_{o} T\right)-1
\end{gathered}
$$

the $c$ and $d$ terms become:

$$
\begin{gathered}
c=\frac{2 \cos \left(2 \omega_{o} T\right)-2 \cos ^{2}\left(\omega_{o} T\right)-\lambda \cos \left(2 \omega_{o} T\right)+\lambda}{2-\lambda} \\
=\frac{\cos \left(2 \omega_{o} T\right)-1-\lambda \cos \left(2 \omega_{o} T\right)+\lambda}{2-\lambda} \\
=\frac{\cos \left(2 \omega_{o} T\right)(1-\lambda)-(1-\lambda)}{2-\lambda} \Rightarrow \\
c=\frac{(1-\lambda)\left(\cos \left(2 \omega_{o} T\right)-1\right)}{2-\lambda} \\
d=\frac{(2-\lambda) \sin \left(2 \omega_{o} T\right)-2 \sin \left(\omega_{o} T\right) \cos \left(\omega_{o} T\right)}{2-\lambda} \\
=\frac{2 \sin \left(2 \omega_{o} T\right)-\lambda \sin \left(2 \omega_{o} T\right)-\sin \left(2 \omega_{o} T\right)}{2-\lambda} \\
=\frac{\sin \left(2 \omega_{o} T\right)-\lambda \sin \left(2 \omega_{o} T\right)}{2-\lambda} \Rightarrow \\
d=\frac{(1-\lambda) \sin \left(2 \omega_{o} T\right)}{2-\lambda}
\end{gathered}
$$


Then

$$
\begin{gathered}
a c=\frac{(1-\lambda)^{2}}{(2-\lambda)}\left(\cos \left(2 \omega_{o} T\right)-1\right)^{2} \\
b d=\frac{(1-\lambda)^{2}}{(2-\lambda)} \sin ^{2}\left(2 \omega_{o} T\right) \\
c^{2}=\frac{(1-\lambda)^{2}}{(2-\lambda)^{2}}\left(\cos \left(2 \omega_{o} T\right)-1\right)^{2} \\
d^{2}=\frac{(1-\lambda)^{2}}{(2-\lambda)^{2}} \sin ^{2}\left(2 \omega_{o} T\right)
\end{gathered}
$$

Using Eqs. (A.14), (A.15), (A.16) and (A.17) the real part of $L$ becomes:

$$
\begin{gathered}
\operatorname{Re}\{L\}=\frac{a c+b d}{c^{2}+d^{2}}=\frac{\frac{(1-\lambda)^{2}}{(2-\lambda)}\left(\left(\cos \left(2 \omega_{o} T\right)-1\right)^{2}+\sin ^{2}\left(2 \omega_{o} T\right)\right)}{\frac{(1-\lambda)^{2}}{(2-\lambda)^{2}}\left(\left(\cos \left(2 \omega_{o} T\right)-1\right)^{2}+\sin ^{2}\left(2 \omega_{o} T\right)\right)} \Rightarrow \\
\operatorname{Re}\{L\}=2-\lambda
\end{gathered}
$$

Appendix 4: Discrete time transfer function coefficients of the control path and disturbance path models.

\begin{tabular}{|c|c|c|c|c|}
\hline & \multicolumn{3}{|c|}{ Control Path Model $\left(G_{c}(z)\right)$} & \multicolumn{2}{c|}{ Disturbance Path Model $\left(G_{d}(z)\right)$} \\
\hline$z^{n}$ & Numerator & Denominator & Numerator & Denominator \\
\hline$z^{0}$ & -0.0256 & 1 & 0.0001 & 1.0000 \\
\hline$z^{-1}$ & 0.2984 & -8.3541 & 0.0000 & -3.0802 \\
\hline$z^{-2}$ & -1.7039 & 34.2693 & -0.0001 & 3.5581 \\
\hline$z^{-3}$ & 6.3122 & -90.6175 & 0.0001 & -1.0666 \\
\hline$z^{-4}$ & -16.9451 & 170.5618 & -0.0000 & -1.6138 \\
\hline$z^{-5}$ & 34.8847 & -237.8488 & -0.0004 & 1.5295 \\
\hline$z^{-6}$ & -56.8627 & 246.4170 & -0.0007 & 0.3176 \\
\hline$z^{-7}$ & 74.7151 & -180.7567 & -0.0006 & -1.1182 \\
\hline$z^{-8}$ & -79.8020 & 74.9743 & 0.0006 & 0.9289 \\
\hline$z^{-9}$ & 69.3235 & 14.1375 & 0.0040 & -1.0742 \\
\hline$z^{-10}$ & -48.6263 & -52.6871 & 0.0058 & 0.7229 \\
\hline$z^{-11}$ & 27.0851 & 47.4996 & 0.0045 & 0.8493 \\
\hline$z^{-12}$ & -11.6187 & -26.2177 & 0.0015 & -2.1715 \\
\hline$z^{-13}$ & 3.6321 & 9.5369 & -0.0012 & 1.9266 \\
\hline$z^{-14}$ & -0.7414 & -2.1444 & -0.0023 & -0.8571 \\
\hline$z^{-15}$ & 0.0744 & 0.2306 & -0.0017 & 0.1576 \\
\hline & & & & \\
\hline
\end{tabular}




\section{References}

Daley S, Hätönen J and Owens DH (2006) Active vibration isolation in a "Smart Spring" mount using a repetitive control approach. IFAC Journal Control Engineering Practice 14(9): 991-997.

Daley S and Zazas I (2012) A recursive least squares based control algorithm for the suppression of tonal disturbances. Journal of Sound and Vibration 331(6): 1270-1290.

Daley S, Zazas I and Hätönen J (2008) Harmonic control of a 'smart spring' machinery vibration isolation system. Proceedings of the Institution of Mechanical Engineers, Part M: Journal of Engineering for the Maritime Environment 222(2):109-119.

Elliott SJ and Nelson PA (1993) Active Noise Control. IEEE Signal Processing Magazine 10(4):12-35.

Elliott SJ, Stothers IM and Nelson PA (1987) A Multiple Error LMS Algorithm and Its Application to the Active Control of Sound and Vibration. IEEE Transactions on Acoustics, Speech, and Signal Processing 35(10), 1423-1987.

Glover JR (1977) Adaptive noise cancelling applied to sinusoidal interferences. IEEE Transactions on Acoustics, Speech, and Signal Processing 25(6):484-491.

Hall SR and Wereley NM (1989) Linear control issues in the higher harmonic control of helicopter vibrations. Proceedings of the 45th Annual Forum of the American Helicopter Society, Boston, MA.

Hätönen J, Daley S, Tammi K and Zazas I (2006) Instantaneous Harmonic Control for Multivariable Systems: Convergence Analysis and Experimental Validation. The Thirteenth International Congress on Sound and Vibration, Vienna, Austria.

Kuo BC (1991) Automatic Control Systems 6th edition. Englewood Cliffs, New Jersey: Prentice-Hall.

Kuo MS and Minjiang J (1996) Passband Disturbance Reduction in Periodic Active noise Control Systems. IEEE Transactions on Speech and Audio Processing 4(2):96-103.

Maciejowski JM (1989) Multivariable Feedback Design. Wokingham, Berkshire, UK, Addison-Wesley.

Mulligan JH (1949) The Effect of Pole and Zero Locations on the Transient Response of Linear Dynamic Systems. Proceedings of the IRE 37(5):516-529.

Orivuori J and Zenger K (2012) Comparison and performance analysis of some active vibration control algorithms. Journal of Vibration and Control 20(1):94-130.

Shaw J, Albion N, Hanker E and Teal R (1989) Higher harmonic control: wind tunnel demonstration of fully effective vibratory hub force suppression. Journal of the American Helicopter Society 34(1):1425.

Sievers LA and Von Flotow AH (1992) Comparison and Extensions of Control Methods for NarrowBand Disturbance Rejection. IEEE Transactions on Signal Processing 40(10):2377-2391.

Widrow B and Stearns SD (1985) Adaptive Signal Processing. Englewood Cliffs, New Jersey, PrenticeHall.

Xu S and Kuo MS (2007) Active Narrowband Noise Control Systems Using Cascading Adaptive Filters. IEEE Transactions on Audio, Speech and Language Processing, 15(2):586-592.

Zazas I (2009) Harmonic Control Algorithms for the Active Control of Periodic Noise and Vibration. $\mathrm{PhD}$ Thesis, University of Sheffield, UK. 